\section{Distribución de la COVID-19 y tuberculosis en la Región Metropolitana de Chile: diferentes enfermedades, similares desigualdades}

\author{
CLAUDIO OLMOS
}

\section{Distribution of COVID-19 and tuberculosis in the Metropolitan Region of Chile: differents diseases, similar inequalities}

Background: Poor living conditions promote the spread of communicable diseases. It happened with Tuberculosis (TB) and is happening with COVID-19. Due to its dynamic nature, this group of diseases must be studied in the context of the social determinants of health. Aim: To describe the epidemiological behavior of COVID-19 in response to the control strategies implemented by the Chilean Ministry of Health and its similarities with the socio-economic distribution of TB in the Metropolitan Region (MR) of Chile. Material and Methods: The 2018 Tuberculosis rates and average income of districts belonging to the MR were described, as well as the incidence rates of COVID-19. A Pearson correlation analysis was applied between the rates of both diseases, to assess similarities in the epidemiological distribution patterns. Results: The RM accounts for over 50\% of the total national cases of COVID-19. After the implementation of selective quarantines, only four districts in the RM managed to control the outbreak (those with the highest incomes). In the rest, a clear increase in cases was observed. The districts with the highest increase in cases were the most disadvantaged, and those with the highest TB rates during 2018, with a correlation coefficient of 0.6 . Conclusions: Control policies cannot deepen health inequalities. If vulnerable groups, as well as the factors that determine their health, are not properly identified, the consequences of the current pandemic could be even more devastating.

(Rev Med Chile 2020; 148: 963-969)

Key words: Coronavirus; Epidemiology; Social Determinants of Health; Tuberculosis.

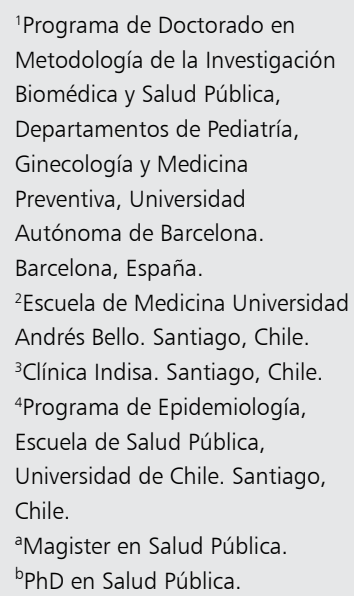

El presente estudio, no contó con ningún tipo de financiamiento.

Recibido el 6 de mayo de 2020 , aceptado el 28 de julio de 2020.

Correspondencia a:

Dra. Valeria Stuardo Á. Programa de Epidemiología,

Escuela de Salud Pública,

Universidad de Chile.

Av. Independencia 939, Santiago,

Chile.

valeria.stuardo@uchile.cl
L a tuberculosis (TB) fue una de las primeras enfermedades infecciosas descritas en la historia de la humanidad, asociada historicamente a la pobreza y rezago social ${ }^{1}$.

A nivel mundial, se estima que 10,0 millones de personas enfermaron de TB en 2018 y cada día más de 4.000 personas mueren a causa de esta enfermedad. En Chile desde 2014 hay un aumento sostenido de la incidencia, alcanzando en 2018 los $14,9 \times 100.000$ hab.
La enfermedad causada por el nuevo coronavirus SARS-Cov-2, se inició a finales de diciembre de 2019 en la ciudad de Wuhan en China, al 4 de mayo de 2020, la OMS ha reportado un total de 3.349.786 casos confirmados de COVID-19 y más de 238.000 fallecidos a nivel mundial ${ }^{2}$.

El mundo ha sido testigo de las consecuencias de una enfermedad que hasta el momento no tiene vacuna. Diversas estrategias de contención de brotes han sido implementadas para disminuir 
la trasmisión viral, entre ellas el confinamiento domiciliario, distanciamiento social, uso de elementos de protección y el lavado de manos frecuente, sin embargo, éstas medidas no son tan fáciles de seguir para muchos grupos vulnerables que viven en comunidades muy densas y/o en viviendas precarias ${ }^{3}$.

Al inicio del brote de COVID-19 en Chile se implementó una estrategia de cuarentena selectiva, la cual consiste en un confinamiento domiciliario diferenciado territorialmente, no homogéneo. En un principio esta estrategia se centró en comunas del sector Oriente de Santiago de Chile (altos ingresos económicos), lo que permitió el control de la transmisión en dicha zona, sin embargo, el virus continuó penetrando en las comunidades más vulnerables, en las cuales la estrategia no fue aplicada ${ }^{4}$.

Las condiciones de vida, la precariedad de la vivienda, el hacinamiento, comorbilidades, abuso de drogas, un nivel educacional deficiente, posición socioeconómica, precarias condiciones de vida y trabajo, propician la propagación de enfermedades transmisibles ${ }^{5,6}$. Producto de su carácter dinámico, este grupo de enfermedades deben ser estudiadas en el contexto de los determinantes sociales de la salud $^{7,8}$.

Se sabe que en contextos de crisis las desigualdades sociales se incrementan, ha ocurrido históricamente con la TB y está ocurriendo ahora con la COVID-19. Por ello, el objetivo de este estudio es decribir la relación entre los patrones epidemiológicos del COVID-19 y la Tuberculosis en la Región Metropolitana de Chile, desde la perspectiva de los determinantes de la salud.

\section{Materiales y Métodos}

Se realizó un análisis de carácter ecológico, para describir el comportamiento epidemiológico de la COVID-19 en respuesta a la estrategia de cuarentena selectiva implementada por el Ministerio de Salud chileno en la Región Metropilitana (RM) y sus similitutes con la distribución de la Tuberculosis.

Se distinguen dos períodos en relación a las medidas de contención de la COVID-19: el primero, desde que se inicia el brote hasta la semana en que se implementa la cuarentena selectiva (Semanas $10^{\mathrm{a}}$ a $13^{\mathrm{a}}$ ), y el segundo, que es durante la implementación de esta estrategia (Semanas $14^{\mathrm{a}}$ a $\left.16^{\mathrm{a}}\right)$.

Se comparararon las tasas de incidencia de COVID-19, de las comunas (unidades territoriales) pertenecientes a los Servicios de Salud Metropolitano Central (SSMC), Norte (SSMN) y Sur (SSMS), además de aquellas incorporadas en la cuarentena selectiva, en total 21 comunas.

Con el objetivo de complementar el análisis se incorporó la variable de ingreso promedio mensual (en USD mensuales) por persona a nivel comunal, como un indicador del Nivel Socioeconómico, información obtenida del Instituto Nacional de Estadísticas de Chile (INE).

Con la información disponible en el Departamento de Epidemiología del MINSAL, se graficó en tres patrones la evolución del número de casos de COVID-19 antes y después del inicio de cuarentenas selectivas, para las 21 comunas estudiadas.

Se realizó una correlación lineal simple (correlación de Pearson) entre las tasa de incidencia de TB del año 2018 y la tasa promedio de COVID-19 durante las semanas epidemiológicas 14, 15 y 16 en las comunas pertenecientes a los SSMC, SSMN y SSMS.

El número de casos de TB fue obtenido desde los registros de vigilancia del Programa de Control y Eliminación de Tuberculosis (PROCET); los casos de COVID-19 de los informes epidemiológicos del Ministerio de Salud de Chile y la población por comuna, del CENSO 2017. El analisis se realizó mediante el software STATA 13.

\section{Resultados}

El brote de COVID-19 se inició en Chile en la semana epidemiológica no 10 del año 2020. Hasta el día 4 de mayo de 2020, la Región Metropolitana registraba 13.528 casos confirmados de COVID-19 (65,5\% del total de casos nacionales).

Los primeros brotes de transmisión comunitaria, se dieron en la zona Oriente de Santiago, las cuales se caracterizan por presentar los mayores ingresos económicos del país. El Gobierno de Chile, decretó cuarentena selectiva para 7 comunas de la Región Metropolitana durante la semana epidemiológica no 13 (26 de marzo). Estas son: Lo Barnechea, Vitacura, Las Condes, Providencia, Nuñoa, Santiago e Independencia. Los criterios para seleccionar aquellas comunas no fueron 
informados. No fue hasta el 30 de marzo, que se liberaron los datos comunales mediante el "Primer Informe Epidemiológico de enfermedad por COVID-19 del Departamento de Epidemiología del Ministerio de Salud".

La Tabla 1, muestra las tasas de incidencia acumulada por COVID-19 en 21 comunas de la Región Metropolitana, en dos períodos: antes y durante la cuarentena (Primer período de la semana $10^{\mathrm{a}}$ a $13^{\mathrm{a}}$ y el segundo período de la semana $14^{\mathrm{a}}$ a 16a). También se incorpora el ingreso promedio por persona en cada comuna, con el fin de brindar una idea de las características socioeconómicas de cada comuna. Obsérvese que en el primer período, tres comunas que no fueron consideradas para la cuarentena selectiva (San Miguel, Huechuraba y San Joaquín) tenían tasas de incidencia de COVID-19 mayores a otras comunas en donde sí se aplico la estrategia.

Por otro lado, se observa que las 4 comunas con mayor aumento de casos acumulados de COVID-19 entre ambos períodos (Pedro Aguirre Cerda, San Joaquín, Estación Central y Recoleta), exhiben un ingreso promedio de USD \$235 por persona, mientras que las únicas 4 comunas que consiguieron una disminución en el número de casos acumulados de COVID-19 después de las cuarentenas (Vitacura, Las Condes, Providencia y Lo Barnechea), tienen un ingreso promedio de USD \$1.191 por persona.

Tabla 1. Tasa de incidencia de casos de COVID-19 según comuna de residencia, e ingreso promedio/mes por persona, Región Metropolitana, 2020

\begin{tabular}{|c|c|c|c|}
\hline \multirow[t]{2}{*}{ Comuna } & \multicolumn{2}{|c|}{ Incidencia Acumulada COVID-19 (x 100 mil) } & \multirow{2}{*}{$\begin{array}{l}\text { Ingresos/mes promedio } \\
\text { por persona (USD) }\end{array}$} \\
\hline & Semanas 10, 11, 12 y 13 & Semanas $14,15,16 * *$ & \\
\hline Vitacura* & 85,8 & 22,7 & $\$ 1.346$ \\
\hline Las Condes * & 54,7 & 22,4 & $\$ 1.338$ \\
\hline Providencia* & 52,0 & 42,5 & $\$ 1.043$ \\
\hline Lo Barnechea* & 54,8 & 52,4 & $\$ 1.039$ \\
\hline Santiago* & 25,2 & 57,8 & $\$ 478$ \\
\hline Huechuraba & 15,1 & 32,9 & $\$ 433$ \\
\hline Cerrillos & 3,4 & 41,6 & $\$ 317$ \\
\hline Ñuñoa* & 26,8 & 51,2 & $\$ 316$ \\
\hline Maipú & 7,4 & 32,7 & $\$ 271$ \\
\hline Recoleta & 8,9 & 47,9 & $\$ 266$ \\
\hline Quilicura & 6,7 & 33,4 & $\$ 260$ \\
\hline San Joaquín & 12,6 & 61,8 & $\$ 255$ \\
\hline San Miguel & 18,8 & 63,9 & $\$ 246$ \\
\hline Estación Central & 7,7 & 39,2 & $\$ 245$ \\
\hline Independencia* & 10,6 & 64,1 & $\$ 235$ \\
\hline Conchalí & 10,1 & 38,1 & $\$ 210$ \\
\hline San Bernardo & 9,6 & 52,6 & $\$ 205$ \\
\hline Lo Espejo & 3,9 & 53,0 & $\$ 201$ \\
\hline La Cisterna & 10,0 & 42,8 & $\$ 198$ \\
\hline El Bosque & 10,5 & 52,3 & $\$ 191$ \\
\hline Pedro Aguirre Cerda & 6,5 & 63,1 & $\$ 173$ \\
\hline
\end{tabular}

Fuente: Décimo Informe Epidemiológico COVID-19, Dpto. de Epidemiología - Ministerio de Salud de Chile. Las tasas aquí exhibidas corresponden al 29 de marzo. *Comunas en cuarentena. ${ }^{* *}$ Semanas cuarentena selectiva. 
Con la información disponible en el Departamento de Epidemiología del MINSAL, fue posible graficar la evolución del número de casos por comuna, antes y después del inicio de cuarente- nas selectivas. De acuerdo a su comportamiento epidemiológico, las 21 comunas fueron agrupadas en tres patrones (Figura 1).

En la Figura 1 se observa que mientras las 4

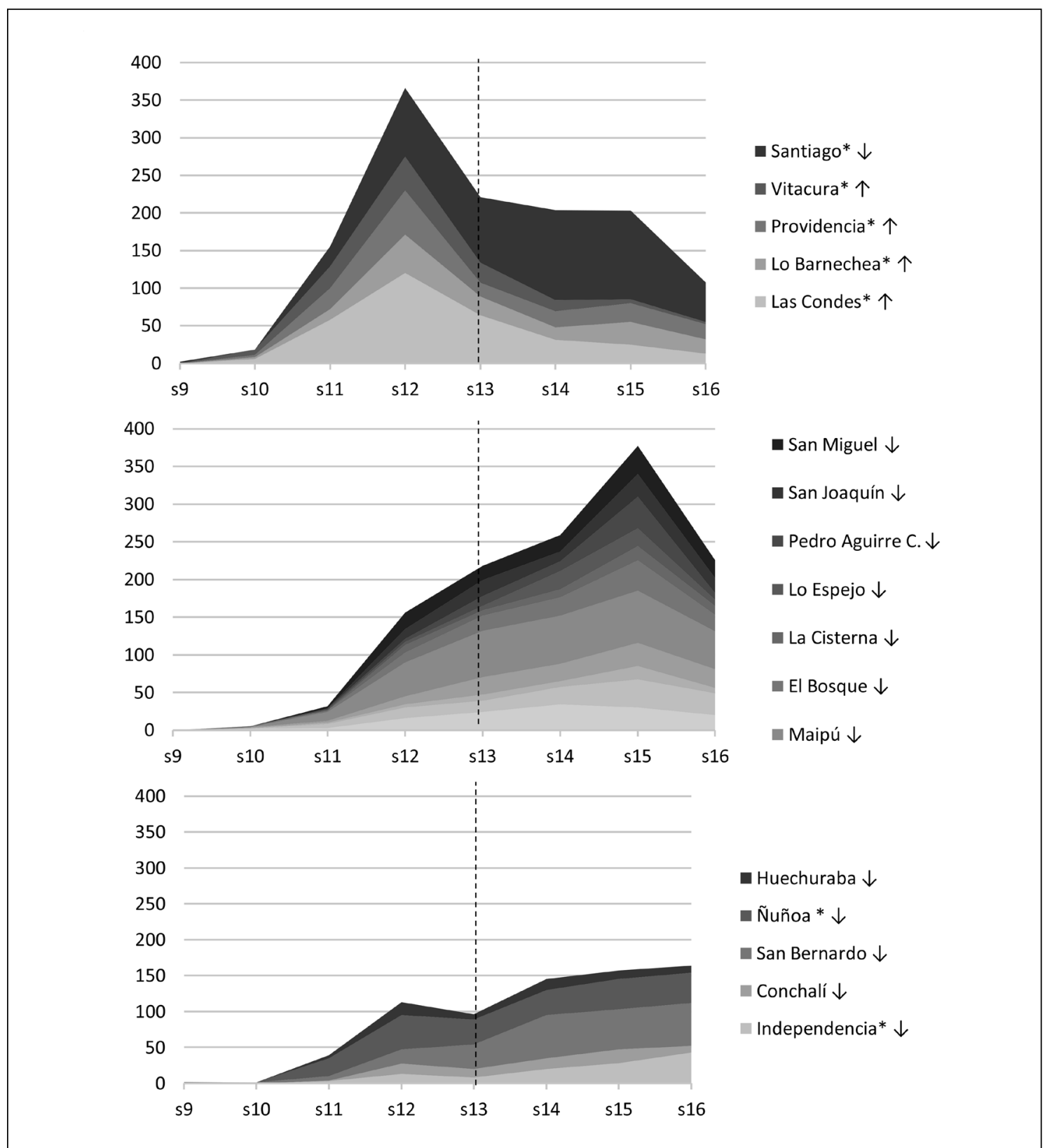

Figura 1. Número de casos de COVID-19, antes y después del inicio de cuarentena selectiva, en comunas de altos y bajos ingresos de la Región Metropolitana de Chile, 2020. Fuente: Creación propia basado en Informe Epidemiológico COVID-19, Dpto. de Epidemiología - Ministerio de Salud de Chile. *Comunas en cuarentena selectiva (Semanas 14 a 16a ). ( $\uparrow$ ): Vitacura, Providencia. Lo Barnechea, Las Condes, comunas con ingresos altos, promedio mensual entre 1.000 y 1.500 USD por persona. $(\downarrow)$ : Comunas con ingresos bajos, promedio mensual entre 100 y 500 USD por persona. 


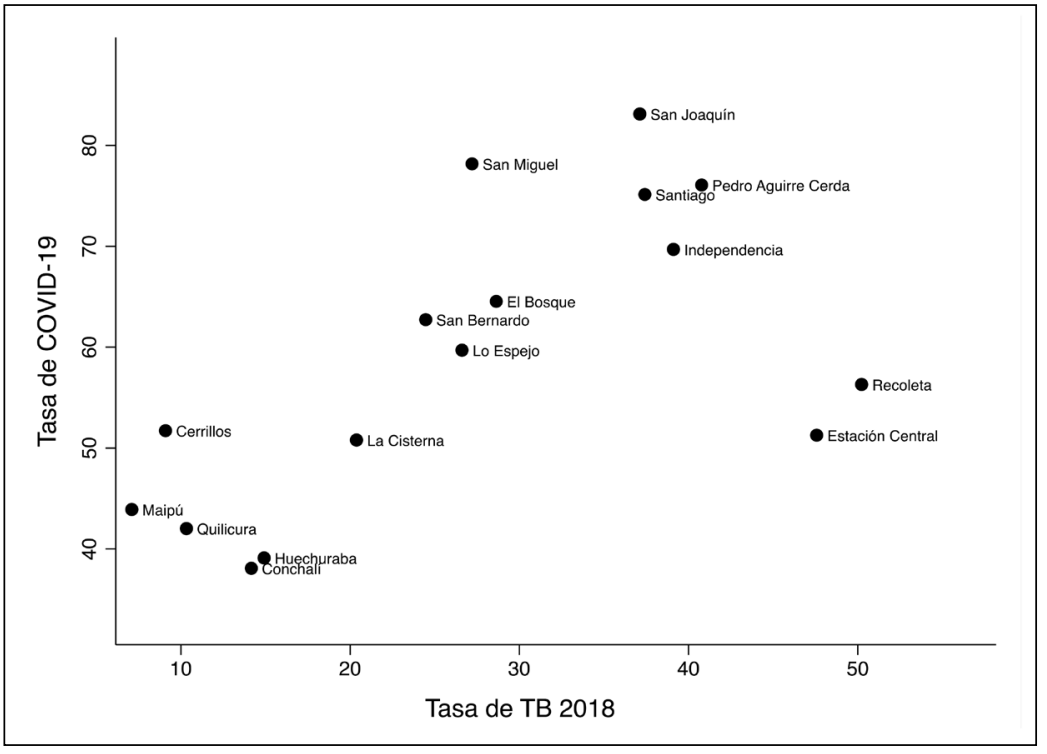

Figura 2. Correlación entre la tasa de incidencia de Tuberculosis 2018 y la tasa de COVID-19, en comunas pertenecientes a los Servicios de Salud Norte, Central y Sur. Fuente: Creación propia de los autores. Coeficiente de correlación $=0,6067$. * La tasa de COVID-19 corresponde a la incidencia acumulada entre el 22 de marzo y 18 de abril de 2020 (últimas 4 semanas epidemiológicas). comunas en cuarentena con mayores ingresos (Providencia, Lo Barnechea, Las Condes y Vitacura), lograron controlar el brote, las comunas de Nuñoa, Independencia y Santiago no lo consiguieron. En el resto de las comunas, que no fueron consideradas en la estrategia de cuarentenas selectivas, se observa un claro aumento de casos.

Las comunas con mayor aumento de casos de COVID-19 entre el primer y segundo período observado, fueron Pedro Aguirre Cerda, San Joaquín, Estación Central y Recoleta. Coincidentemente, las dos primeras fueron las comunas con tasas más altas de TB del SSMS durante el 2018 (40,8 y 37,1 x 100.000 hab., respectivamente), Estación Central fue la comuna con mayor tasa de TB del SSMC (47,6 x 100.000 hab.) y Recoleta, fue la comuna con mayor tasa de TB del SSMN (50,2 x 100.000 hab.).

Al realizar un análisis de correlación simple (Figura 2) entre las tasas de Tuberculosis de 2018 y la tasa de COVID-19 por comunas se observó un grado de correlación alta $(\mathrm{r}=0,6063)$. Las comunas de Recoleta y Estación Central, aparecen como las comunas que se correlacionan en menor medida con sus respectivas tasas de incidencia de COVID-19, sin embargo, esto podría atribuirse a que ambas tienen tasas de incidencia de TB considerablemente más altas que el resto de las comunas. En efecto, cuando se excluye a estas dos comunas del cálculo, el coeficiente de correlación resulta ser de: 0,8812 .

\section{Discusión y Conclusiones}

El inicio de la epidemia de COVID-19 en Chile deja de manifiesto las profundas desigualdades sociales presentes en el país. Los resultados de este trabajo, dejan en evidencia que, a pesar de que el concepto de vulnerabilidad es dinámico, las crisis sanitarias y humanitarias incrementan estas desigualdades sociales y visibilizan las falencias estructurales de los estados?.

Los determinantes sociales de la salud, persistentemente están presentes a la hora de comprender el comportamiento de la enfermedad y los grupos de mayor riesgo.

Pareciera que las epidemias revelan ciertas divisiones culturales o fronteras invisibles; aquellos que pertenecen a un nivel socioeconómico alto, están más protegidos tanto por sus condiciones de vida, como por su capacidad de acceder servicios de salud, mientras que las poblaciones más vulnerables, carecen precisamente de aquello ${ }^{10,11}$.

Son muchos los factores socioculturales, económicos y políticos que inciden en el riesgo de las personas, pero las limitaciones y condiciones de vida, que deben enfrentar determinados grupos, 
como los adultos mayores o migrantes, los sitúa como grupos especialmente vulnerables ${ }^{12-17}$.

Aunque los primeros casos de COVID-19 en Chile se hayan presentado en las áreas más favorecidas, el virus termina asentándose en los barrios más vulnerables. Y en ese contexto, resulta esencial evaluar el efecto que tuvo la implementación de la estrategia de cuarentenas selectivas y la pertinencia de su continuidad. Como se afirmaba en una reciente editorial, una persona que no es considerada vulnerable en el comienzo de una pandemia puede volverse vulnerable de acuerdo a las políticas que se implementen ${ }^{11}$.

Es probable que los determinantes sociales implicados históricamente en la propagación de enfermedades asociadas a la pobreza y rezago social, como lo es la TB, sean los mismos que actualmente se están asociando a la propagación de COVID-19 18,19 .

A la luz de la evidencia, era ciertamente predecible que las mayores tasas de COVID-19 se concentrarían en comunas con mayor vulnerabilidad social, mayores índices de hacinamiento, y menores niveles educacionales y socioeconómicos.

Al hacer frente al COVID-19, a la Tuberculosis o cualquier otra enfermedad trasmisible, los responsables de generar políticas de control, deberían asegurarse de no profundizar las desigualdades en salud. Si los grupos vulnerables, así como los factores que determinan su salud, no se identifican adecuadamente, las consecuencias de la actual pandemia podrían ser aún más devastadoras.

La fortaleza de un sistema de salud es inseparable de los sistemas sociales más amplios que lo rodean ${ }^{20}$. La protección de la salud se basa no solo en un sistema de salud que funcione bien con cobertura universal, sino también en la inclusión social, la justicia y la solidaridad. En ausencia de estos factores, las desigualdades se magnifican y persisten en el tiempo.

\section{Referencias}

1. Báguena Cervellera MJ. La tuberculosis en la historia. Rev Acad Med Comunitat Valencia 2012; 12: 1-8.

2. World Health Organization (WHO). Coronavirus disease 2019 (COVID-19) Situation Report-104. World Heal Organ [Internet] 2020;(April):2633. Available from: https://www.who.int/emergencies/diseases/novel-coronavirus-2019

3. Ryan BJ, Coppola D, Canyon DV, Brickhouse M,
Swienton R. COVID-19 Community Stabilization and Sustainability Framework: An Integration of the Maslow Hierarchy of Needs and Social Determinants of Health. Disaster Med Public Health Prep [Internet] 2020; 1-16. Available from: http://www.ncbi.nlm.nih.gov/pubmed/32314954.

4. Ministerio de Salud. Informe Epidemiológico Enfermedad por COVID-19, 30 de marzo de 2020. 2020; Available from: https://cdn.digital.gob.cl/public_files/ Campañas/Corona-Virus/Reportes/INFORME_EPI_ COVID19_20200330.pdf.

5. Chingonzoh R, Manesen MR, Madlavu MJ, Sopiseka N, Nokwe M, Emwerem M, et al. Risk factors for mortality among adults registered on the routine drug resistant tuberculosis reporting database in the Eastern Cape Province, South Africa, 2011 to 2013. PLoS One 2018; 13 (8): 1-15.

6. Gayoso R, Dalcolmo M, Braga JU, Barreira D. Predictors of mortality in multidrug-resistant tuberculosis patients from Brazilian reference centers, 2005 to 2012. Brazilian J Infect Dis 2018; 22 (4): 305-10.

7. Semenza JC, Suk JE, Tsolova S. Social determinants of infectious diseases: a public health priority 2010; 15 (27).

8. Braveman P. Accumulating knowledge on the social determinants of health and infectious disease. Public Health Rep 2011; 126 (Suppl. 3): 28-30.

9. Crispi F, Cherla A, Vivaldi EA, Mossialos E. Rebuilding the broken health contract in Chile. Lancet [Internet]. 2020; 395 (10233): 1342. Available from: http://dx.doi. org/10.1016/S0140-6736(20)30228-2.

10. Penman-Aguilar A, Talih M, Huang D, Moonesinghe R, Bouye K, Beckles G. Measurement of Health Disparities, Health Inequities, and Social Determinants of Health to Support the Advancement of Health Equity. J Public Heal Manag Pr 2016; 22 (1): 33-42.

11. Editorial The Lancet. Racism and discrimination in COVID-19 responses 2020; 395 (April): 1194.

12. Pareek M, Greenaway C, Noori T, Munoz J, Zenner D, Toms C, et al. The impact of migration on tuberculosis epidemiology and control in high-income countries: a review. BMC Med 2016; 14 (1): 48.

13. Bernales M, Cabieses B, McIntyre AM, Chepo M, Flaño J, Obach A. Determinantes sociales de la salud de niños migrantes internacionales en Chile: evidencia cualitativa. Salud Publica Mex 2018; 60 (5, sep-oct): 566.

14. Cabral JF, da Silva AMC, Mattos IE, Neves Á de Q, Luz LL, Ferreira DB, et al. Vulnerability and associated factors among older people using the family health strategy. Cienc e Saude Coletiva 2019; 24 (9): 3227-36.

15. Sarvimäki A, Stenbock-hult B. Abstract Background: 
Objective: Research design: Participants and research context: Ethical considerations: Findings: Discussion and conclusion: 2016;

16. Santelli JS, Bazaale JM, Gray RH, Olawore O, Serwadda D, Grabowski MK, et al. Migration and risk of HIV acquisition in Rakai, Uganda: a population-based cohort study. Lancet HIV [Internet] 2018; 5 (4): e1819. Available from: http://dx.doi.org/10.1016/S23523018(18)30009-2.

17. Hayward S, Harding RM, McShane H, Tanner R. Factors influencing the higher incidence of tuberculosis among migrants and ethnic minorities in the UK. F1000Research 2018; 7 (0): 461.
18. Hargreaves JR, Boccia D, Evans CA, Adato M, Petticrew $\mathrm{M}$, Porter JDH. The social determinants of tuberculosis: from evidence to action. Am J Public Health 2011; 101 (4): 654-62.

19. Tomás BA, Pell C, Cavanillas AB, Solvas JG, Pool R, Roura M. Tuberculosis in migrant populations. A systematic review of the qualitative literature. PLoS One 2013; 8 (12): 1-12.

20. Organización Panamericana de la Salud. Determinantes sociales de la salud en la Región de las Américas. Salud en las Américas [Internet] 2011; 1-9. Available from: https://www.paho.org/salud-en-las-americas-2017/?post_t_es=determinantes-sociales-de-la-salud\&lang=es. 